\title{
EMPTY CONVEX 5-GONS IN PLANAR POINT SETS
}

\author{
SEONG-YOON AnN AND Ensil KAnG*
}

\begin{abstract}
Erdös posed the problem of determining the minimum number $g(n)$ such that any set of $g(n)$ points in general position in the plane contains an empty convex $n$-gon. In 1978, Harborth proved that $g(5)=10$. We reprove the result in a geometric approach.
\end{abstract}

\section{Introduction}

Due to the importance in computer graphics, geometric modellings, motion planing, and other areas, the study of the convex polygons has a renewed interest, recently. In this paper, we reprove a known result related to the Erdös and Szekeres problem which is a classic problem counting empty convex $k$-gons in planar points sets.

In 1935, Erdös and Szekeres posed the problem of determining the smallest positive integer $f(n)$ so that any set of at least $f(n)$ points in opened position in the plane contains a convex $n$-gon and also conjectured that $f(n)=2^{n-2}+1$ for all $n \geq 3[1]$. Until now, the problem has been solved for the values $n=3,4$, and 5 only ([4]). For larger values of $n$, the best known upper bound $f(n) \leq\left(\begin{array}{c}2 n-5 \\ n-2\end{array}\right)+1$ was recently proved by Tóth and Valtr[7]. Later, Erdös posed a similar problem on empty convex polygons, which is of determining a smallest positive integer $g(n)$ such that any set of at least $g(n)$ points in general position in the plane contains an empty convex $n$-gon. For $n=3$ and 4 , it is easy to show that $g(3)=3$ and $g(4)=5$. For $n=5$, Harborth proved that $g(5)=10[2]$. But Horton showed that $g(n)$ does not exist for all $n \geq 7[3]$. The only open case is for $n=6$. Even the existence of $g(6)$ still

Received July 3, 2009. Accepted August 5, 2009.

2000 Mathematics Subject Classification. Primary 52C10.

Key words : Combinatorial geometry, convex polygon.

This study was supported by research funds from Chosun University, 2005.

*Corresponding author. E-mail : ekang@chosun.ac.kr. 
remains as a question. Here we reprove that $g(5)=10$ by visualizing the method of Harborth.

Theorem 1.1. Any set $X$ of at least 10 points in general position in the plane contains the vertex set of an empty convex pentagon.

\section{Proof of Theorem 1}

Let $X$ be a set of $n \geq 10$ points in general position in the plane. Since $f(5)=9$, among the points in $X$, there are 5 points in convex position.

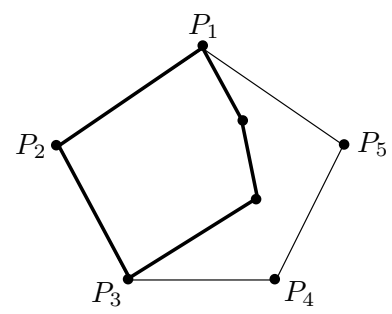

Figure 1.

If there are $m \geq 2$ points of $X$ inside the convex pentagon, we construct another pentagon with two of $m$ points inside the pentagon and three of 5 points on the pentagon. The pentagon reconstructed has points inside less than $m$. In this way, we reduce the number of points inside a pentagon and eventually will get a convex pentagon $P$ with zero or one point inside it. If $P$ is non-empty, denote the points of $X$ on $P$ by $P_{1}, P_{2}, P_{3}, P_{4}, P_{5}$ in a counterclockwise order and the point inside $P$ by $C$. If $C$ is inside one of triangles $\Delta P_{1} P_{2} P_{3}, \Delta P_{2} P_{3} P_{4}, \Delta P_{3} P_{4} P_{5}$, $\Delta P_{4} P_{5} P_{1}$, and $\Delta P_{5} P_{1} P_{2}$, then we can construct an empty convex pentagon with $C$ and four of five points $P_{1}, P_{2}, P_{3}, P_{4}, P_{5}$.

Suppose that $C$ is not inside either of the triangles and so is in the small pentagon inside $P$ (see the figure 2). We divide cases according to place of points outside $P$ and will get an empty convex pentagon from $C, P_{1}, P_{2}, P_{3}, P_{4}, P_{5}$ and points outside $P$. We denote $\left(P_{i}, P_{j}\right)$ the open half-plane lying to the left of the line $\overline{P_{i} P_{j}}$ directed from $P_{i}$ to $P_{j}$. Then we consider the region $R_{i}=\left(C, P_{i}\right) \cap\left(P_{i+1}, P_{i}\right) \cap\left(P_{i+1}, P_{i+2}\right)$, $i=1,2,3,4,5$ and $P_{6}=P_{1}$ and $P_{7}=P_{2}$ (see the figure 3 ). 


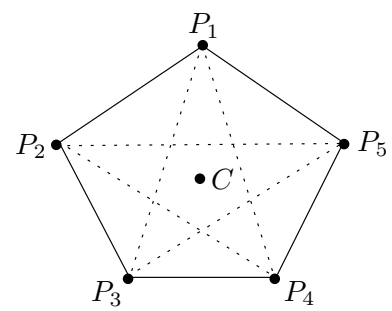

Figure 2.

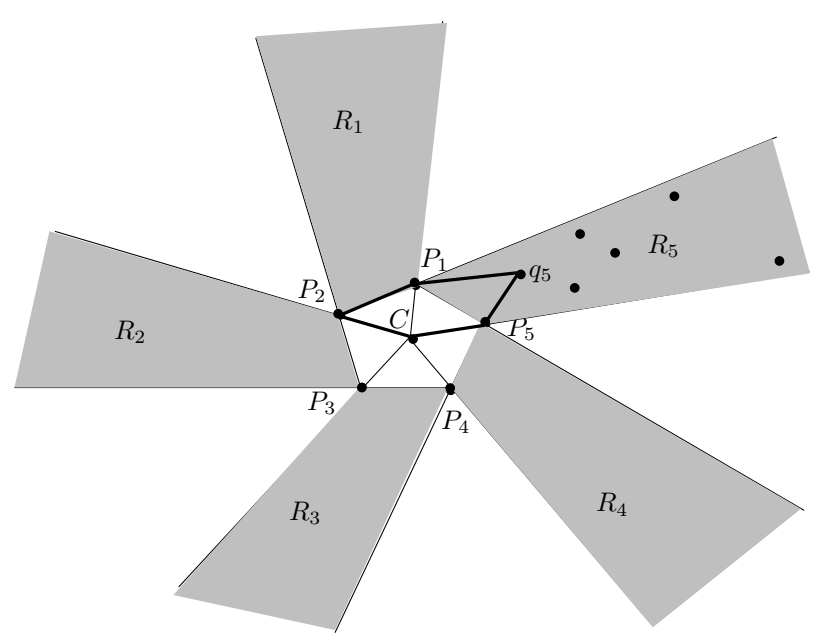

FIGURE 3.

If there is a point of $X$ in the region $R_{i}$, then we can construct an empty convex pentagon with $C$ and $P_{i}, P_{i+1}, P_{i+2}$ and the innermost one $q_{5}$ of points in $R_{5}$. Similarly, for the region $R_{i}^{\prime}=\left(P_{i}, C\right) \cap\left(P_{i}, P_{i-1}\right) \cap$ $\left(P_{i-2}, P_{i-1}\right), i=1,2,3,4,5$ and $P_{0}=P_{5}$ and $P_{-1}=P_{4}$, we can also get an empty convex pentagon in the case that there is a point in the region $R_{i}^{\prime}$ (see the figure 4 ).

Now we suppose that there are no points of $X$ in either $R_{i}$ or $R_{i}^{\prime}$, $i=1,2,3,4,5$. Then at least four points of $X$ are in the region $S=$ $S_{1} \cup S_{2} \cup S_{3} \cup S_{4} \cup S_{5}$, where $S_{i}=\left(P_{i}, P_{i-1}\right) \cap\left(P_{i+2}, P_{i+1}\right), i=1,2,3,4,5$ and $P_{0}=P_{5}, P_{6}=P_{1}$, and $P_{7}=P_{2}$ (see the figure 5). We first consider the case that each region of $S_{i}, i=1,2,3,4,5$, has at most one point. In this case, there are three consecutive regions $S_{i}, S_{i+1}, S_{i+2}$ each of 


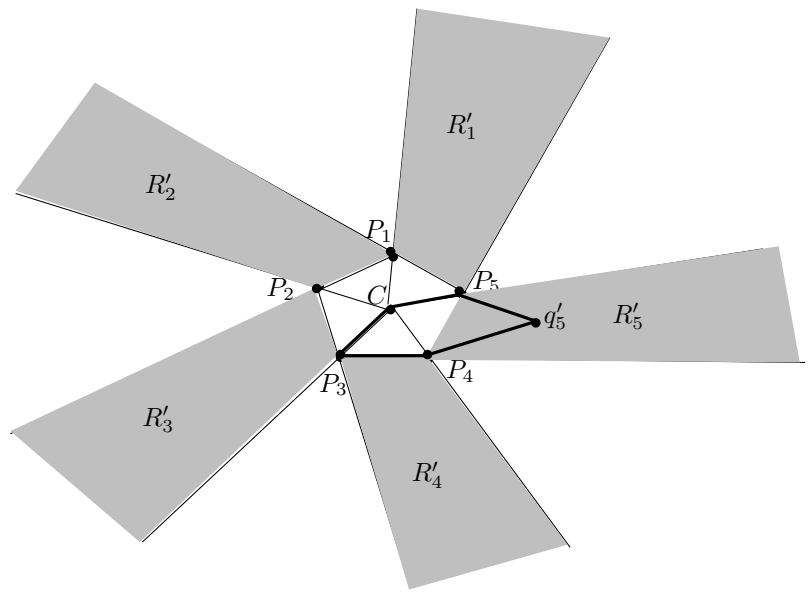

FIGURE 4.

which has one point of $X$, and so that the three points of $S_{i} \cup S_{i+1} \cup S_{i+2}$ with $P_{i+1}$ and $P_{i+2}$ form an empty convex pentagon (see the figure 5).

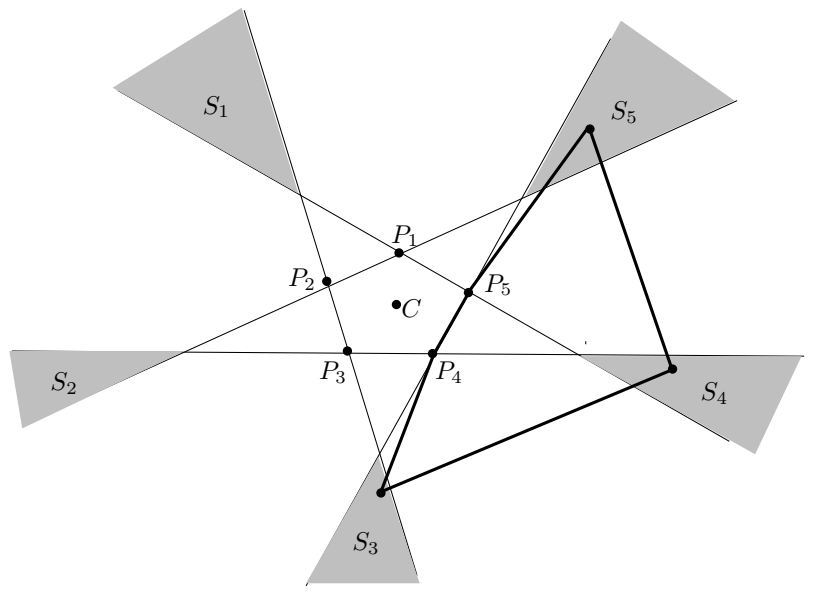

FiguRE 5 .

Now we suppose that there are two or more points in a region $S_{i}$. We may assume the region as $S_{5}$. Let $v_{1}$ be the innermost point of $X$ in $S_{5}$ so that there is no point of $X$ inside the triangle $\Delta v_{1} P_{1} P_{5}$. Here we divide two cases according to the position of the points of $X$ remaining in $S_{5}$. 
Case 1. There are points of $X$ in the region $T_{0}=\left(\left(P_{5}, v_{1}\right) \cup\right.$ $\left.\left(v_{1}, P_{1}\right)\right) \cap S_{5}$, then choose the innermost one $t_{0}$ so that $v_{1}, t_{0}, P_{1}, C$, $P_{5}$ form an empty convex pentagon (see the figure 6).

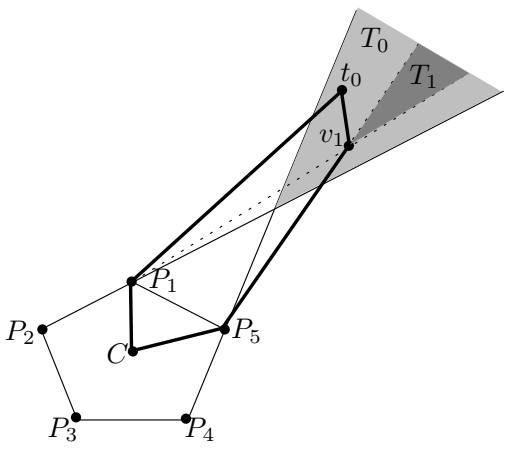

FiguRE 6.

Case 2. If there is no point of $X$ in $T_{0}$, the region $T_{1}=\left(P_{1}, v_{1}\right) \cap$ $\left(v_{1}, P_{5}\right)$ has at least one point of $X$. Choose the innermost one $t_{1}$.

Case 2.1. If there is a point of $X$ in $S_{1} \cup S_{2}$, we can get an empty convex pentagon with $t_{1}, v_{1}, P_{1}, P_{2}$ and one point of $S_{1} \cup S_{2}$.

Case 2.2. If there is a point of $X$ in $S_{3} \cup S_{4}$, we can get an empty convex pentagon with $t_{1}, v_{1}, P_{5}, P_{4}$ and one point of $S_{3} \cup S_{4}$ (see the figure 7$)$.

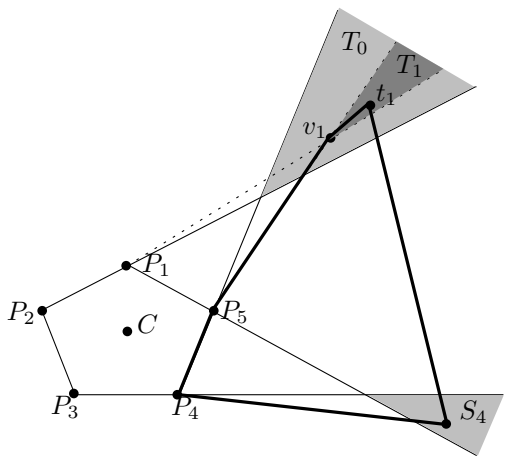

FiguRE 7. 
Case 2.3. Suppose that there is no point in $S_{1} \cup S_{2} \cup S_{3} \cup S_{4}$. Then there is at least two more points of $X$ in $T_{1}$. Choose the innermost one $t_{2}$ of $X$ in $T_{1}$ except for $t_{1}$. If $t_{2}$ is in $\left(t_{1}, v_{1}\right)$, then $t_{2}, t_{1}, v_{1}, P_{5}, P_{4}$ form an empty convex pentagon. Otherwise, $t_{2}, t_{1}, v_{1}, P_{1}, P_{2}$ form an empty convex pentagon(see the figure 8).

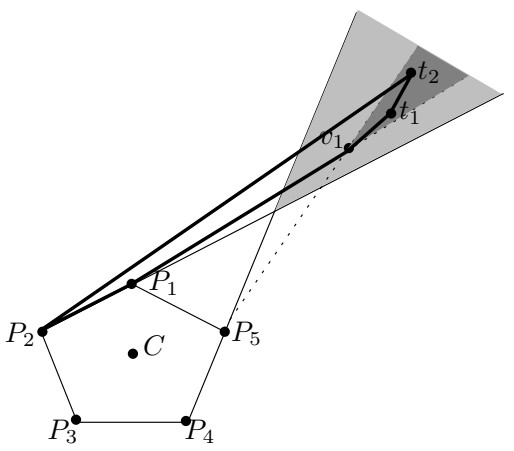

FIGURE 8.

\section{References}

[1] P. Erdös, G. Szekeres, A combinatorial problem in geometry, Compositio Math. 2 (1935), 463-470

[2] H. Harborth, Konvexe Fünfecke in ebenen Punktmengen, Elem. Math. 33 (1978), 116-118

[3] J.D. Horton, Sets with no empty convex 7-gons, Canad. Math. Bull. 26 (1983), $482-484$

[4] J.D. Kalbfleisch, J.G. Kalbfleisch, R.G. Stanton, A combinatorial problem on convex regions, Proc. Louisiana Conf. Combinatorics, Graph Theory and Computing, Louisiana State Univ., Baton Rouge, La, 1970. Congr. Number 1. (1970), 180-188

[5] W. Morris, V. Soltan, The Erdös-Szekeres problem on points in convex position - A survey, Bull. Amer. Math. Soc. 37 (2000), 437-458

[6] G. Töth, P. Valtr, Note on the Erdös-Szekeres Theorem, Discrete Comput. Geom. 19 (1998), 457-459

[7] G. Toth, P. Valtr, The Erdos-Szekeres theorem: upper bounds and related results, Combinatorial and Computational Geometry, 557-568. Math. Sci. Res. Inst. Publ. 52, Cambridge Univ. Press, Cambridge, 2005 
Department of Mathematics,

Graduate School of Education,

Chosun University,

Gwangju 501-759, Korea

Department of mathematics,

College of Natural Sciences,

Chosun University,

Gwangju 501-759, Korea 